\title{
On a New Model Based on Third-Order Nonlinear Multisingular Functional Differential Equations
}

\author{
Zulqurnain Sabir, ${ }^{1}$ Hatıra Günerhan, ${ }^{2}$ and Juan L. G. Guirao ${ }^{3}{ }^{3}$ \\ ${ }^{1}$ Department of Mathematics and Statistics, Hazara University, Mansehra, Pakistan \\ ${ }^{2}$ Mathematics Department, Faculty of Education, Kafkas University, Kars, Turkey \\ ${ }^{3}$ Departamento de Matemática Aplicaday Estadística, Universidad Politécnica de Cartagena, Cartagena 30203, Spain \\ Correspondence should be addressed to Juan L. G. Guirao; juan.garcia@upct.es
}

Received 18 December 2019; Accepted 29 January 2020; Published 28 February 2020

Guest Editor: Praveen Agarwal

Copyright (C) 2020 Zulqurnain Sabir et al. This is an open access article distributed under the Creative Commons Attribution License, which permits unrestricted use, distribution, and reproduction in any medium, provided the original work is properly cited.

\begin{abstract}
In this study, a novel mathematical model based on third-order nonlinear multisingular functional differential equations (MSFDEs) is presented. The designed model is solved by using a well-known differential transformation (DT) scheme that is a very credible tool for solving the nonlinear third-order nonlinear MS-FDEs. In order to check the exactness, efficacy, and convergence of the scheme, some numerical examples are presented based on nonlinear third-order MS-FDEs and numerically solved by using DT scheme. The scheme of differential transformation allows us to find a complete solution and a closed approximate solution of the differential equation. The distinctive advantage of the computational technique is to deal with the complex and monotonous physical problems that are obtained in various branches of engineering and natural sciences. Moreover, a comparison of the obtained numerical outcomes from the exact solutions shows the correctness, accurateness, and exactness of the designed model as well as the presented scheme.
\end{abstract}

\section{Introduction}

The singular study along with functional differential equations (FDEs) is considered very significant for the researcher's community, and the implementations of the FDEs have been noticed in the sixth decade of the nineteen century. The FDEs have a huge variety of applications in many fields; to mention few of them are, models of population growth [1], electrodynamics [2], infection models of HIV-1 [3], models of tumor growth [4], models based on chemical kinetics [5], B-virus infection hepatitis models [6], models of the gene regulations [7], and models of viral infections [8], and many more [9-14]. The singular study based on the differential models is very interesting, complicated, experimental, and challenging for the researchers due to the singularity appearance at origin. There are many singular models in the literature; one of the famous models is Lane-Emden that represents singularity at $x=0$ always. The model of the Lane-Emden is famous as its historic point of view and has been applied broadly due to its huge important and significant applications in the fields of science and technology. Some of the important applications of the Lane-Emden model are that it is used in various phenomena of mathematical physics structure and in the study of astrophysics, such as models of the stellar structure [15], study of thermal explosions model [16], study of the model of isothermal gas spheres [17], oscillating magnetic fields [18], and thermionic currents [19].

In recent decades, the research community is interested to solve the singular nonlinear FDEs numerically due to the singularity and functionality in differential equations. For example, to present the solutions of these nonlinear FDEs, Kadalbajoo and Sharma $[20,21]$ applied a numerical scheme. In order to solve differential-difference based model, Mirzaee and Hoseini [22] implemented a numerical collocation scheme. $\mathrm{Xu}$ and Jin [23] explained the singularly functional perturbed differential model by applying the fractional steps and boundary functions. Geng et al [24] applied a numerical 
approach for the delay differential equations on the basis of singularly perturbed model.

The purpose of the recent study is to present the model based on the nonlinear multisingular (MS) functional differential equations, i.e., MS-FDEs of order three. The modeled form of the third-order nonlinear MS-FDE along with its initial conditions (ICs) is written as

$$
\left\{y^{\prime \prime \prime}\left(x+\tau_{1}\right)+\frac{\alpha}{x} y^{\prime \prime}\left(x+\tau_{2}\right)+\frac{\beta}{x^{2}} y^{\prime}\left(x+\tau_{3}\right)+x y\left(x+\tau_{4}\right)=f(x), \quad y(0)=a, y^{\prime}(0)=b, y^{\prime \prime}(0)=c .\right.
$$

The parameters $\tau_{i}(i=1,2,3,4)$ and $\alpha, \beta, a, b$, and $c$ are the real constant values.

The idea of the above model is achieved by extending the work of Sabir et al. [25] that is used to explain the nonlinear singular FDEs of second order. For the verification and correctness of the designed MS-FDEs model, three different examples have been modeled and numerically solved by using the well-known differential transformation (DT) scheme, and the obtained numerical outcomes of DT scheme are compared with the exact solutions. The DT scheme has been applied to solve many stiff, nonstiff, singular, nonsingular, linear, and nonlinear types of problems. Zhou [26], for the first time, presented the idea of the DT scheme at the end of the $19^{\text {th }}$ century to solve the linear/nonlinear initial value problems based on the analysis of electrical circuit. The DT scheme is basically a numerical approach, which works on the basis of the expansion of TS, which constitutes a polynomial form of the analytic results. The quality of the numerical DT scheme is to require less work and does not require linearization as well as assumptions. This numerical scheme is designed on the basis of an analytical solution by using the polynomial expressions, such as the Taylor series (TS) expansion. But its procedure is more easier than the conventional higher-order TS scheme, which achieves symbolic computation of the necessary derivatives using the data-based functions. Three explanatory and illustrative examples based on model (1) are provided to show the efficacy of the obtained results from the DT scheme. These numerical outcomes are compared with the exact solutions that indicate the proficiency of the designed model as well as the proposed scheme.

Some major key factors of the present study are summarized as follows:

The mathematical modeled form of the third-order nonlinear MS-FDEs is presented successfully by extending the work of Sabir et al. [25]

The designed nonlinear MS-FDEs based on the designed model are addressed numerically by using the famous DT scheme.

Manipulation of the present scheme is to apply the brilliance-obtained outcomes for nonlinear MSFDEs with better precision and outstanding consistency.

The reliability and correctness of the designed model are authentic through the comparison of the numerical results obtained by the DT scheme and the exact results. The overlapping of these results indicates perfection, excellence, and faultlessness for the model.

The third-order functional differential model given in equation (1) is not easy to solve because of nonlinearity, multisingularity, functionality, and harder in nature. DT scheme is the best choice and good selection to handle these types of complicated and complex models.

The rest of the paper is described as follows: the designed detailed methodology on the basis of DT scheme is provided in Section 2. Results and discussion are provided in Section 3. Conclusion along with future research direction is provided in the last section.

\section{Methodology (Differential Transform Scheme)}

The mathematical definition of DT scheme using $y(\zeta)$ is given as

$$
Y(k)=\frac{1}{k !}\left[\frac{\mathrm{d}^{k} y(\zeta)}{\mathrm{d} \zeta^{k}}\right]_{x=0}
$$

The original function in the above equation (2) is $y(\zeta)$, whereas the transformed function (TF) is denoted by $\mathbf{Y}(\mathbf{k})$, which is also called the T-function. The inverse of DT scheme of $\mathbf{Y}(\mathbf{k})$ is provided as

$$
y(\zeta)=\sum_{k=0}^{\infty} Y(k)\left(\zeta-\zeta_{0}\right)^{k} \equiv D^{-1} Y(k)
$$

Using the results of equations (2) and (3), the obtained function becomes

$$
y(\zeta)=\sum_{k=0}^{\infty} \frac{\zeta^{k}}{k !}\left[\frac{\mathrm{d}^{k} y(\zeta)}{\mathrm{d} \zeta^{k}}\right]_{x=0}
$$

Equation (4) provides the concepts of DT scheme that are derived from the TS expansion, and this scheme has not been applied for symbolical assessment of the derivatives. Moreover, comparative derivative values are achieved by using the iterative procedure, which is defined by the transformed original function. In this study, the lowercase and uppercase letters are used to show the original function and the TF, respectively. Using the nature of the above two equations, one can easily prove the TFs have the basic mathematical values provided in Table 1 .

In real applications, $y(\zeta)$ is obtained by a finite series and equation (3) can be described as 
TABle 1: The essential operations of DT scheme.

\begin{tabular}{lc}
\hline Unique function & TF \\
\hline $\begin{array}{l}y(\zeta)=u(\zeta) \pm v(\zeta) \\
y(\zeta)=c u(\zeta)\end{array}$ & $Y(k)=U(k) \pm V(k)$ \\
$y(\zeta)=d^{m} u(\zeta) / d \zeta^{m}$ & $Y(k)=c U(k)$ \\
$y(\zeta)=u(c \zeta)$ & $Y+1)+(k+2) \cdots(k+m) U(k+m)$ \\
$y(\zeta)=u(\zeta / c)$ & $Y(k)=c^{k} U(k)$ \\
$y(\zeta)=d^{m} / d \zeta^{m} u(c \zeta)$ & $Y(k)=U(k) / c^{k}$
\end{tabular}

Equation (5) shows the term $\sum_{k=m+1}^{\infty} \zeta^{k} Y(k)$, which is very small and can be neglected, while $\mathbf{m}$ shows the convergence of natural frequency.

For better explanation of the DT scheme, some important theorems are presented as follows:

Theorem 1. If $f(\zeta)=m(\zeta) o(\zeta)$, then $F(K)=M(k) \otimes O(k)=$ $\sum_{l=0}^{k} M(l) O(k-l)$ (here, $\otimes$ denotes the convolution):

$$
\begin{aligned}
f(\zeta)= & \sum_{k=0}^{\infty} M(k)\left(\zeta-\zeta_{0}\right)^{k} \times \sum_{k=0}^{\infty} O(k)\left(\zeta-\zeta_{0}\right)^{k} \\
= & \left(M(0)+M(1)\left(\zeta-\zeta_{0}\right)+M(2)\left(\zeta-\zeta_{0}\right)^{2}+\cdots\right) \\
= & M(0) O(0)+M(U(0) O(1)+M(1) O(0))\left(\zeta-\zeta_{0}\right) \\
& \times\left(O(0)+O(1)\left(\zeta-\zeta_{0}\right)+O(2)\left(\zeta-\zeta_{0}\right)^{2}+\cdots\right) \\
& +(M(0) O(2)+M(1) O(1)+M(2) O(0))\left(\zeta-\zeta_{0}\right)^{2}+\cdots \\
= & \sum_{k=0}^{\infty} \sum_{l=0}^{k} M(l) O(k-l)\left(\zeta-\zeta_{0}\right)^{k} .
\end{aligned}
$$

By using equation (3), we get

$$
F(K)=\sum_{l=0}^{k} M(l) O(k-l)
$$

Theorem 2. If $f(\zeta)=\zeta^{\phi}$, then
Proof. By using equation (2), we have

$$
F(h)=\left.\frac{1}{h !} \frac{\partial\left(\zeta^{\phi}\right)}{\partial \zeta^{h}}\right|_{t=0}= \begin{cases}\frac{1}{h !} \frac{\partial^{h}\left(\zeta^{h}\right)}{\partial \zeta^{h}}=\frac{h !}{h !}=1, & h=\phi, \\ \frac{1}{h !} \frac{\partial^{h}\left(\zeta^{\phi}\right)}{\partial \zeta^{h}}=0, & h \neq \phi .\end{cases}
$$

Theorem 3. By taking $f(\zeta)=e^{\zeta+c}$, we have $F(k)=e^{c} / k$ !.

Proof. Using equation (2), we get

$$
F(k)=\left.\frac{1}{k !} \frac{\partial\left(e^{\zeta+c}\right)}{\partial \zeta^{k}}\right|_{t=0}=\left.e^{c}\left(\frac{\partial e^{k}}{\partial \zeta^{k}}\right)\right|_{t=0}=\frac{e^{c}}{k !} .
$$

Theorem 4. If $f(\zeta)=q(\zeta+m)$, then [27]

$$
F(k)=\sum_{i=k}^{N}(m)^{i-k}\left(\begin{array}{l}
i \\
k
\end{array}\right) Q(i) \text { for } N \longrightarrow \infty .
$$

Proof. By using differential inverse transform of $Y(k)$, we have

$$
\begin{aligned}
f(\zeta)= & \sum_{l=2}^{\infty} Q(k)\left(\zeta-\zeta_{0}+m\right)^{k}=Q(0)+Q(1)\left(\left(\zeta-\zeta_{0}\right)+m\right) \\
& +Q(2)\left(\left(\zeta-\zeta_{0}\right)+m\right)^{2}+Q(3)\left(\left(\zeta-\zeta_{0}\right)+m\right)^{3}+\cdots \\
= & Q(0)+Q(1)\left(\zeta-\zeta_{0}\right)+Q(1) m+Q(2)\left(\zeta-\zeta_{0}\right)^{2}+Q(2) m^{2} \\
& +2 Q(2)\left(\zeta-\zeta_{0}\right) m+Q(3) m^{3}+3 Q(3)\left(\zeta-\zeta_{0}\right) m^{2}+3 Q(3)\left(\zeta-\zeta_{0}\right)^{2} m+Q(3)\left(\zeta-\zeta_{0}\right)^{3}+\cdots
\end{aligned}
$$




$$
\begin{aligned}
= & \left(Q(0)+Q(1) m+Q(2) m^{2}+Q(3) m^{3}+\cdots\right) \\
& +\left(\zeta-\zeta_{0}\right)\left(Q(1)+2 Q(2) m+3 Q(3) m^{2}+\cdots\right) \\
& +\left(x-x_{0}\right)^{2}(Q(2)+3 Q(3) m+\cdots)+\left(\zeta-\zeta_{0}\right)^{3}(Q(3)+\cdots)+\cdots \\
= & \sum_{l=0}^{\infty} \frac{l !}{0 !(l-0) !} m^{l-0} Q(l)+\sum_{l=0}^{\infty} \frac{l !}{1 !(l-1) !} m^{l-1} Q(l)\left(\zeta-\zeta_{0}\right) \\
& +\sum_{l=2}^{\infty} \frac{l !}{2 !(l-2) !} m^{l-2} Q(l)\left(\zeta-\zeta_{0}\right)^{2}+\sum_{l=0}^{\infty} \frac{l !}{3 !(l-3) !} m^{l-3} Q(l)\left(\zeta-\zeta_{0}\right)^{3} \\
& +\cdots+\sum_{l=0}^{\infty} \frac{l !}{k !(l-k) !} m^{l-k} Q(l)\left(\zeta-\zeta_{0}\right)^{k} \\
& =\sum_{k=0}^{\infty} \sum_{l=k}^{\infty} \frac{l !}{k !(l-k) !} m^{l-k} Q(l)\left(\zeta-\zeta_{0}\right)^{k}=\sum_{k=0}^{\infty} \sum_{l=k}^{\infty}\left(\begin{array}{l}
l \\
k
\end{array}\right) m^{l-k} Q(l)\left(\zeta-\zeta_{0}\right)^{k} .
\end{aligned}
$$

By comparing equations (3) and (12), $Y(k)$ becomes

$$
F(k)=\sum_{l=k}^{N}\left(\begin{array}{l}
l \\
k
\end{array}\right) m^{l-k} Q(l) \text { for } N \longrightarrow \infty .
$$

Theorem 5. If $y(\zeta)=d^{b} u(\zeta+m) / d \zeta^{b}$, then

$$
Y(h)=\frac{(h+b) !}{h !} \sum_{l=h+b}^{N}(m)^{l-h-b}\left(\begin{array}{c}
l \\
h+b
\end{array}\right) U(l) \text { for } N \longrightarrow \infty .
$$

Proof. Suppose $y(\zeta)=u(\zeta+m)$, in equation (2), we have

$$
Y(h)=\frac{1}{h !} \frac{\partial^{h}}{\partial \zeta^{h}}\left(\frac{d^{b} y(\zeta)}{d \zeta^{b}}\right)=\frac{(h+b) !}{h !} Y(h+b) .
$$

Using the $Y(k)$ values from equation (13), it becomes

$$
\begin{aligned}
\frac{(h+b) !}{h !} Y(h+b) & =\frac{(h+b) !}{h !}\left(\frac{d^{h+b}\left(\sum_{l=h}^{N}\left(\begin{array}{l}
l \\
h
\end{array}\right) m^{l-k} U(l)\right)}{d \zeta^{b}}\right) \\
& =\frac{(h+b) !}{h !} \sum_{l=h+b}^{N}\left(\begin{array}{c}
l \\
h+b
\end{array}\right) m^{l-(h+b)} U(l), \\
Y(h) & =\frac{1}{h !} \frac{\partial^{h}}{\partial \zeta^{h}}\left(\frac{d^{b} y(\zeta)}{d \zeta^{b}}\right)=\frac{1}{h !} \frac{\partial^{h}}{\partial \zeta^{h}}\left(\frac{d^{b} u(\zeta+m)}{d \zeta^{b}}\right) \\
& =\frac{(h+b) !}{h !} \sum_{l=h+b}^{N}\left(\begin{array}{c}
l \\
h+b
\end{array}\right) m^{l-h-b} U(l),
\end{aligned}
$$

$N \longrightarrow \infty$.

\section{Results and Discussion}

To present the numerical solutions based on the designed third-order nonlinear MS-FDE model, the nonlinear study is very important and many investigations have been provided in references [28-33]. Three different examples have been presented, and the solutions of the examples are performed by using the DT scheme.

Example 1. Consider the nonlinear third-order MS-FDE given as

$$
\left\{\begin{array}{l}
y^{\prime \prime}(\psi-1)+\frac{1}{\psi} y^{\prime \prime}(\psi+1)+\frac{2}{\psi^{2}} y^{\prime}(\psi+2)+\psi y(\psi)=e^{\psi-1} \\
+\frac{1}{\psi} e^{\psi+1}+\frac{2}{\psi^{2}} e^{\psi+2}+\psi e^{\psi} \\
y(0)=1 \\
y^{\prime}(0)=1 \\
y^{\prime \prime}(0)=1 .
\end{array}\right.
$$

Multiplied by $\psi^{2}$, the achieved form is given as

$$
\begin{aligned}
& \psi^{2} y^{\prime \prime \prime}(\psi-1)+\psi y^{\prime \prime}(\psi+1)+2 y^{\prime}(\psi+2)+\psi^{3} y(\psi) \\
& =\psi^{2} e^{\psi-1}+\psi e^{\psi+1}+2 e^{\psi+2}+\psi^{3} e^{\psi},
\end{aligned}
$$

The DT scheme is applied to solve the model given in equation (17). By using the definitions of one-dimensional DT and the corresponding transformation of equation (17), the obtained system becomes 


$$
\begin{aligned}
\delta(k-2) \otimes(k+3)(k+2)(k+1) & \sum_{\sigma=k+3}^{N}(-1)^{\sigma-k-3}\left(\begin{array}{c}
\sigma \\
k+3
\end{array}\right) Y(\sigma) \\
& +\delta(k-1) \otimes(k+2)(k+1) \sum_{\sigma=k+2}^{N}(1)^{\sigma-k-2}\left(\begin{array}{c}
\sigma \\
k+2
\end{array}\right) Y(\sigma) \\
& +2(k+1) \sum_{\sigma=k+1}^{N}(2)^{\sigma-k-1}\left(\begin{array}{c}
\sigma \\
k+1
\end{array}\right) Y(\sigma)+\delta(k-3) \otimes Y(k) \\
= & \delta(k-2) \otimes \frac{1}{k !} e^{-1}+\delta(k-1) \otimes \frac{1}{k !} e+2 \frac{1}{k !} e^{2}+\delta(k-3) \otimes \frac{1}{k !}
\end{aligned}
$$

Theorem 1 is used in equation (19), we get

$$
\begin{aligned}
\sum_{\eta=0}^{k} \sum_{i=\eta+3}^{N} \delta(k-\eta-2)(\eta+3)(\eta+2)(\eta+1)(-1)^{i-\eta-3}\left(\begin{array}{c}
\sigma \\
\eta+3
\end{array}\right) Y(\sigma) \\
\quad+\sum_{\eta=0}^{k} \sum_{\sigma=v+2}^{N} \delta(k-\eta-1)(\eta+2)(\eta+1)\left(\begin{array}{c}
\sigma \\
\eta+2
\end{array}\right) Y(\sigma) \\
\quad+2(k+1) \sum_{i=k+1}^{N}(2)^{\sigma-k-1}\left(\begin{array}{c}
\sigma \\
k+1
\end{array}\right) Y(\sigma) \\
\quad+\sum_{\eta=0}^{k} \delta(\eta-3) Y(k-\eta) \\
=\sum_{\eta=0}^{k} \delta(k-\eta-2) \frac{1}{\eta !} e^{-1}+\sum_{\eta=0}^{k} \delta(k-\eta-1) \frac{1}{\eta !} e+2 \frac{1}{k !} e^{2} \\
\quad+\sum_{\eta=0}^{k} \delta(k-\eta-3) \frac{1}{\eta !}
\end{aligned}
$$

Using the ICs given in equation (17), we have

$$
\begin{aligned}
& Y(0)=1, \\
& Y(1)=1, \\
& Y(2)=\frac{1}{2} .
\end{aligned}
$$

Taking $N=4$ and $k=0$ and 1 , by using equations (20) and (21), the obtained linear algebraic equation system is written as

$$
\left\{\begin{array}{l}
12 Y(3)+32 Y(4)+3=e^{2}, \\
30 Y(3)+108 Y(4)+3=e+2 e^{2} .
\end{array}\right.
$$
have

By solving the above coupled equations given in (22), we

$$
\begin{aligned}
& y(3)=\frac{\left(-57-8 e+11 e^{2}\right)}{84}, \\
& y(4)=\frac{\left(9+2 e-e^{2}\right)}{56} .
\end{aligned}
$$

By using the values of $Y(k)$ for $k=0$ and 1 in $y(\psi)$, i.e., the inverse-reduced DT, the results are written as

$$
\begin{aligned}
y(\psi)= & \sum_{k=0}^{\infty} Y(k) \psi^{k}=1+\psi+\frac{1}{2} \psi^{2}+\frac{\left(-57-8 e+11 e^{2}\right)}{84} \psi^{3} \\
& +\frac{\left(9+2 e-e^{2}\right)}{56} \psi^{4}+O\left(\psi^{5}\right) .
\end{aligned}
$$

Repeat the process by using the equations (20) and (25) for $N=6$ and $k=0,1$, and 2 . The solution of the obtained linear algebraic equations system is given as

$$
\begin{aligned}
& y(3)=\frac{\left(-57-8 e+11 e^{2}\right)}{84}, \\
& y(4)=\frac{\left(9+2 e-e^{2}\right)}{56}, \\
& y(5)=\frac{128 e^{2}-128 e-495}{17120}, \\
& y(6)=\frac{-8 e^{2}+8 e+51}{5136} .
\end{aligned}
$$

By using the inverse-reduced DT $y(k)$, the solutions will be as follows:

$$
\begin{aligned}
y(\psi)= & \sum_{k=0}^{\infty} Y(k) \psi^{k}=1+\psi+\frac{1}{2} \psi^{2}+\frac{\left(-57-8 e+11 e^{2}\right)}{84} \psi^{3} \\
& +\frac{\left(9+2 e-e^{2}\right)}{56} \psi^{4} \\
& +\frac{128 e^{2}-128 e-495}{17120} \psi^{5}+\frac{-8 e^{2}+8 e+51}{5136} \psi^{6}+O\left(\psi^{7}\right) .
\end{aligned}
$$

Table 2 shows the comparison of the present numerical results for $N=4$ and $N=6$ with the exact solutions. The $y(\psi)$ results are slightly varied by changing the $N$ parameter values. It is clear in Table 2 that the proposed and exact solutions overlapped each other.

Example 2. Consider the third-order MS-FDEs with its ICs:

$$
\left\{\begin{array}{l}
y^{\prime \prime \prime}(\psi-1)+\frac{1}{\psi} y^{\prime \prime}(\psi+1)+\frac{2}{\psi^{2}} y^{\prime}(\psi+2)+\psi y(\psi)=\psi^{5} \\
+45 \psi+48+\frac{108}{\psi}+\frac{64}{\psi^{2}} \\
y(0)=1 \\
y^{\prime}(0)=0 \\
y^{\prime \prime}(0)=0 .
\end{array}\right.
$$

Equation (27) becomes 
TABLE 2: Comparison of the obtained results and exact solutions for $N=4$ and $N=6$.

\begin{tabular}{lccc}
\hline$\psi$ & DT $(N=4)$ & DT $(N=6)$ & Exact solution \\
\hline 0.01 & 1.01005 & 1.01005 & 1.01005 \\
0.02 & 1.02020 & 1.02020 & 1.02020 \\
0.03 & 1.03045 & 1.03045 & 1.03045 \\
0.04 & 1.04080 & 1.04080 & 1.04081 \\
0.05 & 1.05125 & 1.05125 & 1.05127 \\
0.06 & 1.06180 & 1.06180 & 1.06183 \\
0.07 & 1.07246 & 1.07246 & 1.07250 \\
0.08 & 1.08322 & 1.09408 & 1.08328 \\
0.09 & 1.09408 & 1.09408 & 1.09417 \\
0.1 & 1.10504 & 1.10504 & 1.10517 \\
0.2 & 1.22044 & 1.22044 & 1.22140 \\
0.3 & 1.34683 & 1.34685 & 1.34985 \\
0.4 & 1.48515 & 1.48522 & 1.49182 \\
0.5 & 1.63663 & 1.63686 & 1.64872 \\
0.6 & 1.80282 & 1.80341 & 1.82211 \\
0.7 & 1.98556 & 1.98688 & 2.01375 \\
0.8 & 2.18698 & 2.18965 & 2.22554 \\
0.9 & 2.40955 & 2.41451 & 2.45960 \\
\hline
\end{tabular}

$$
\begin{array}{r}
\psi^{2} y^{\prime \prime \prime}(\psi-1)+\psi y^{\prime \prime}(\psi+1)+2 y^{\prime}(\psi+2)+\psi^{3} y(\psi) \\
=\psi^{7}+45 \psi^{3}+48 \psi^{2}+108 \psi+64 .
\end{array}
$$

The definition of one-dimensional DT scheme is applied and taking the consistent transform of equation (27), the system is given as

$$
\begin{gathered}
\delta(k-2) \otimes(k+3)(k+2)(k+1) \sum_{\sigma=k+3}^{N}(-1)^{\sigma-k-3}\left(\begin{array}{c}
\sigma \\
k+3
\end{array}\right) Y(\sigma) \\
+\delta(k-1) \otimes(k+2)(k+1) \sum_{\sigma=k+2}^{N}(1)^{\sigma-k-2}\left(\begin{array}{c}
\sigma \\
k+2
\end{array}\right) Y(\sigma) \\
\quad+2(k+1) \sum_{\sigma=k+1}^{N}(2)^{\sigma-k-1}\left(\begin{array}{c}
\sigma \\
k+1
\end{array}\right) Y(\sigma)+\delta(k-3) \otimes Y(k) \\
=\delta(k-7)+45 \delta(k-3)+48 \delta(k-2)+108 \delta(k-1)+64 \delta(k) .
\end{gathered}
$$

Appling Theorem 1, we have

$$
\begin{aligned}
\sum_{\eta=0}^{k} \sum_{\sigma=\eta+3}^{N} \delta(k-\eta-2)(\eta+3)(\eta+2)(\eta+1)(-1)^{\sigma-\eta-3}\left(\begin{array}{c}
\sigma \\
\eta+3
\end{array}\right) Y(\sigma) \\
+\sum_{\eta=0}^{k} \sum_{\sigma=\eta+2}^{N} \delta(k-\eta-1)(\eta+2)(\eta+1)\left(\begin{array}{c}
\sigma \\
l+2
\end{array}\right) Y(\sigma) \\
\quad+2(k+1) \sum_{\sigma=k+1}^{N}(2)^{\sigma-k-1}\left(\begin{array}{c}
\sigma \\
k+1
\end{array}\right) Y(\sigma)+\sum_{\eta=0}^{k} \delta(\eta-3) Y(k-\eta) \\
=\delta(k-7)+45 \delta(k-3)+48 \delta(k-2)+108 \delta(k-1)+64 \delta(k) .
\end{aligned}
$$

Using the ICs given in equation (27), we have

$$
\begin{aligned}
& Y(0)=1, \\
& Y(1)=0, \\
& Y(2)=0 .
\end{aligned}
$$

Taking the values of $N=4$ and $k=0$ and 1 in equations (30) and (31), the obtained linear algebraic equations system is given as

$$
\left\{\begin{array}{l}
12 Y(3)+32 Y(4)=32, \\
30 Y(3)+108 Y(4)=108 .
\end{array}\right.
$$
have

Solving the coupled equations given in system (32), we

$$
\begin{aligned}
& y(3)=0, \\
& y(4)=1 .
\end{aligned}
$$

Using the $Y(k)$ values for $k=0$ and 1 in the inversereduced DT $y(\psi)$, the obtained results are given as

$$
y(\psi)=\sum_{k=0}^{\infty} Y(k) \psi^{k}=1+\psi^{4} .
$$

Repeat the same procedure using equations (30) and (31), for $N=5$ and $k=0,1$, and 2. The following linear algebraic system becomes

$$
\left\{\begin{array}{l}
30 Y(3)+108 Y(4)+340 Y(5)=108, \\
12 Y(3)+32 Y(4)+80 Y(5)=32, \\
3 Y(3)+8 Y(4)+60 Y(5)=8 .
\end{array}\right.
$$

By solving the equation system, we have

$$
\begin{aligned}
& y(3)=0, \\
& y(4)=1, \\
& y(5)=0 .
\end{aligned}
$$

By using the inverse-reduced DT $y(k)$, the solutions will be as follows:

$$
y(\psi)=\sum_{k=0}^{\infty} Y(k) \psi^{k}=1+\psi^{4} .
$$

This is the exact solution of the Example 2.

Example 3. Consider the third-order MS-FDEs

$$
\left\{\begin{array}{l}
y^{\prime \prime \prime}(\psi-1)+\frac{1}{\psi} y^{\prime \prime}(\psi+1)+\frac{2}{x^{2}} y^{\prime}(\psi+2)+\psi y(\psi)=\psi^{4} \\
+\psi+18+\frac{30}{\psi}+\frac{24}{\psi^{2}} \\
y(0)=1, \\
y^{\prime}(0)=0 \\
y^{\prime \prime}(0)=0 .
\end{array}\right.
$$

Multiplying by $\psi^{2}$, equation (38) takes the form as

$$
\begin{aligned}
& \left.\psi^{2} y^{\prime \prime \prime}(\psi-1)+\psi y^{\prime \prime}(\psi+1)\right) 2 y^{\prime}(\psi+2)+\psi^{3} y(\psi) \\
& =\psi^{6}+\psi^{3}+18 \psi^{2}+30 \psi+24 .
\end{aligned}
$$

Using the definitions of the one-dimensional DT scheme, we get 


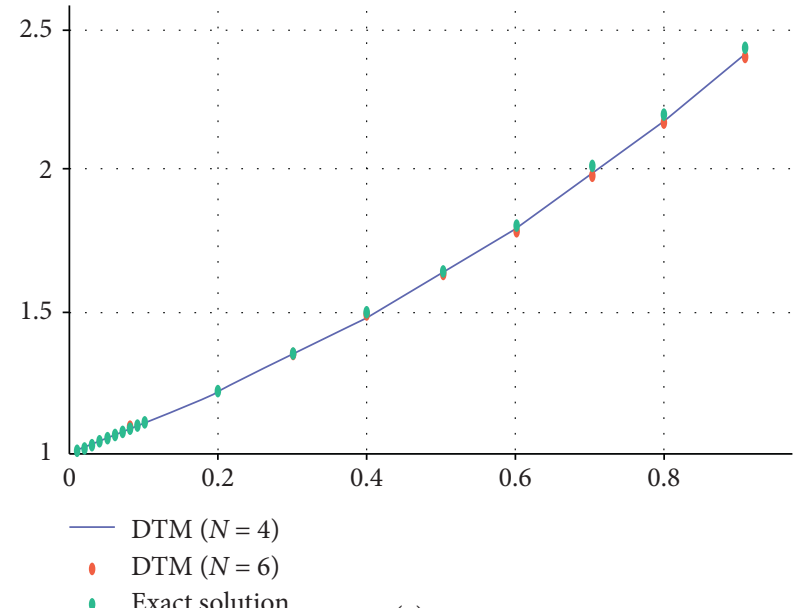

(a)

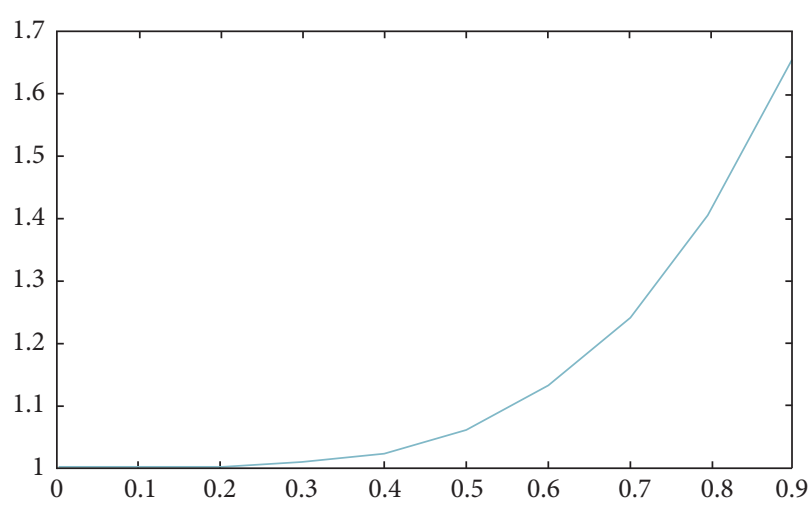

(b)

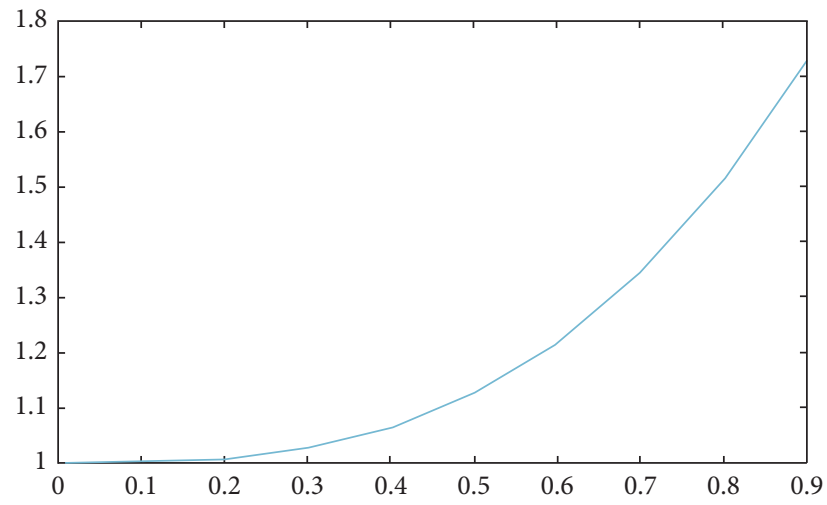

(c)

Figure 1: Plots of $y(\psi)$ for Examples $1-3$ in the range of $0.01<\psi<0.9$. Comparison of numerical results of $y(\psi)$ for (a) Example 1, (b) Example 2, and (c) Example 3.

$$
\begin{array}{r}
\delta(k-2) \otimes(k+3)(k+2)(k+1) \sum_{\sigma=k+3}^{N}(-1)^{\sigma-k-3}\left(\begin{array}{c}
\sigma \\
k+3
\end{array}\right) Y(\sigma) \\
+\delta(k-1) \otimes(k+2)(k+1) \sum_{\sigma=k+2}^{N}(1)^{\sigma-k-2}\left(\begin{array}{c}
\sigma \\
k+2
\end{array}\right) Y(\sigma) \\
+2(k+1) \sum_{\sigma=k+1}^{N}(2)^{\sigma-k-1}\left(\begin{array}{c}
\sigma \\
k+1
\end{array}\right) Y(\sigma)+\delta(k-3) \otimes Y(k) \\
=\delta(k-6)+\delta(k-3)+18 \delta(k-2)+30 \delta(k-1)+24 \delta(k) .
\end{array}
$$

By using Theorem 1, we get

$$
\begin{aligned}
\sum_{\eta=0}^{k} \sum_{\sigma=\eta+3}^{N} \delta(k-\eta-2)(\eta+3)(\eta+2)(\eta+1)(-1)^{\sigma-l-3}\left(\begin{array}{c}
\sigma \\
\eta+3
\end{array}\right) Y(\sigma) \\
\quad+\sum_{\eta=0}^{k} \sum_{\sigma=\eta+2}^{N} \delta(k-\eta-1)(\eta+2)(\eta+1)\left(\begin{array}{c}
\sigma \\
\eta+2
\end{array}\right) Y(\sigma) \\
\quad+2(k+1) \sum_{\sigma=k+1}^{N}(2)^{\sigma-k-1}\left(\begin{array}{c}
\sigma \\
k+1
\end{array}\right) Y(\sigma)+\sum_{\eta=0}^{k} \delta(\eta-3) Y(k-\eta) \\
=\delta(k-6)+\delta(k-3)+18 \delta(k-2)+30 \delta(k-1)+24 \delta(k) .
\end{aligned}
$$

Using the ICs of equation (38), we have

$$
\begin{aligned}
& Y(0)=1, \\
& Y(1)=0, \\
& Y(2)=0 .
\end{aligned}
$$

Taking $N=4$ and $k=0$ and 1 in equations (41) and (42), the linear algebraic equations system is achieved as

$$
\left\{\begin{array}{l}
12 Y(3)+32 Y(4)=12, \\
30 Y(3)+108 Y(4)=30 .
\end{array}\right.
$$

Solving the above system, we get

$$
\begin{aligned}
& y(3)=1, \\
& y(4)=0 .
\end{aligned}
$$

Using the values of $Y(k)$ for $k=0$ and 1 into the inverse-reduced DT of $y(\psi)$, the solution will be as follows:

$$
y(\psi)=\sum_{k=0}^{\infty} Y(k) \psi^{k}=1+\psi^{3} .
$$

Repeat the same process for $N=5$ and $k=0,1$, and 2 by using the above equations. The obtained linear algebraic equation system is given as 


$$
\left\{\begin{array}{l}
30 Y(3)+108 Y(4)+340 Y(5)=30 \\
12 Y(3)+32 Y(4)+80 Y(5)=12 \\
3 Y(3)+8 Y(4)+60 Y(5)=3
\end{array}\right.
$$

The solution of the obtained system of equations is

$$
\begin{aligned}
& y(3)=1, \\
& y(4)=0, \\
& y(5)=0 .
\end{aligned}
$$

By using the inverse-reduced DT of $y(k)$, the solutions becomes

$$
y(\psi)=\sum_{k=0}^{\infty} Y(k) \psi^{k}=1+\psi^{3} .
$$

which is the exact solution. $y(\psi)$ is calculated for different values of $N$ and shown in Figure 1(c).

For more clear understanding, Figure 1 is plotted that has been drawn between 0.01 and 0.9. The values of $N$ are taken as 4 and 6 . One can see that the exact and present solutions for $N=4$ and $N=6$ are overlapped to each other in the range of 0.01 to 0.09 . However, by increasing a slight value in the step size, the results are slightly different but accurate. So it can be concluded that small step size gives more accurate values as compared to large step size.

\section{Conclusion}

The task to model the third-order MS-FDEs is very difficult to handle as well as construct the differential equations of the designed model. The numerical differential transformation scheme is applied successfully to check the correctness and the accurateness of the designed model. The traditional/ conventional techniques fail to solve such multisingular, nonlinear, functionality, and harder nature models. The numerical differential transformation scheme is a good choice to solve such types of complicated, nonlinear, and multisingular models. Consequently, the adopted scheme is effective as well as suitable too. The present study shows that the DTM is an effective and suitable technique to solve such types of equations that we have investigated here. The comparison of the exact and solutions obtained from the differential transformation scheme has also been presented in tabular form as well as graphically. The overlapping of the results shows the perfection of the designed model and establishes the worth of the designed scheme. However, it is observed that when the step size is small, the results are more accurate, but making a slight increase in the step size, the results are overlapped and the error is reduced.

In future, a system of third-order and fourth-order multisingular functional models will be modeled and it will be verified by using the differential transformation scheme as well as famous artificial neural networks [34-39].

\section{Data Availability}

No data were used to support this study.

\section{Conflicts of Interest}

The authors declare that there are no conflicts of interest regarding the publication of this paper.

\section{Acknowledgments}

The first author was partially supported by the Ministerio de Ciencia, Innovación y Universidades under grant no. PGC2018-097198-B-I00 and Fundación Séneca de la Región de Murcia under grant no. 20783/PI/18.

\section{References}

[1] X. Liu and G. Ballinger, "Boundedness for impulsive delay differential equations and applications to population growth models," Nonlinear Analysis: Theory, Methods \& Applications, vol. 53, no. 7-8, pp. 1041-1062, 2003.

[2] M. Dehghan and F. Shakeri, "The use of the decomposition procedure of Adomian for solving a delay differential equation arising in electrodynamics," Physica Scripta, vol. 78, no. 6, Article ID 065004, 2008.

[3] P. W. Nelson and A. S. Perelson, "Mathematical analysis of delay differential equation models of HIV-1 infection," Mathematical Biosciences, vol. 179, no. 1, pp. 73-94, 2002.

[4] M. Villasana and A. Radunskaya, "A delay differential equation model for tumor growth," Journal of Mathematical Biology, vol. 47, no. 3, pp. 270-294, 2003.

[5] M. R. Roussel, "The use of delay differential equations in chemical kinetics," The Journal of Physical Chemistry, vol. 100, no. 20, pp. 8323-8330, 1996.

[6] S. A. Gourley, Y. Kuang, and J. D. Nagy, "Dynamics of a delay differential equation model of hepatitis B virus infection," Journal of Biological Dynamics, vol. 2, no. 2, pp. 140-153, 2008.

[7] D. Bratsun, D. Volfson, L. S. Tsimring, and J. Hasty, "Delayinduced stochastic oscillations in gene regulation," Proceedings of the National Academy of Sciences, vol. 102, no. 41, pp. 14593-14598, 2005.

[8] G. Huang, Y. Takeuchi, and W. Ma, "Lyapunov functionals for delay differential equations model of viral infections," SIAM Journal on Applied Mathematics, vol. 70, no. 7, pp. 2693-2708, 2010.

[9] D. Baleanu, S. Suganya, M. M. Arjunan, and M. Nagaraj, "Approximate controllability of second-order nonlocal impulsive functional integro-differential systems in Banach spaces," Korea Mathematical Society, vol. 55, no. 4, pp. 1065-1092, 2018.

[10] D. Baleanu and P. Agarwal, "On generalized fractional integral operators and the generalized Gauss hypergeometric functions," Abstract and Applied Analysis, vol. 2014, Article ID 630840, 5 pages, 2014.

[11] A. Shvets and A. Makaseyev, "Deterministic chaos in pendulum systems with delay," Applied Mathematics and Nonlinear Sciences, vol. 4, no. 1, pp. 1-8, 2019.

[12] J. M. Sanz-Serna and B. Zhu, "Word-series high-order averaging of highly oscillatory differential equations with delay," 2019, http://arxiv.org/abs/1906.06944.

[13] V. Klinshov, O. Maslennikov, and V. Nekorkin, "Jittering regimes of two spiking oscillators with delayed coupling," Applied Mathematics and Nonlinear Sciences, vol. 1, no. 1, pp. 197-206, 2016.

[14] T. Caraballo, M. A. Diop, and A. Mane, "Controllability for neutral stochastic functional integrodifferential equations 
with infinite delay," Applied Mathematics and Nonlinear Sciences, vol. 1, no. 2, pp. 493-506, 2016.

[15] A.-M. Wazwaz, "A new method for solving singular initial value problems in the second-order ordinary differential equations," Applied Mathematics and Computation, vol. 128, no. 1, pp. 45-57, 2002.

[16] W. M. Abd-Elhameed, E. H. Doha, A. S. Saad, and M. A. Bassuony, "New galerkin operational matrices for solving lane-Emden type equations," Revista mexicana de astronomía y astrofísica, vol. 52, no. 1, pp. 83-92, 2016.

[17] K. Boubaker and R. A. Van Gorder, "Application of the BPES to Lane-Emden equations governing polytropic and isothermal gas spheres," New Astronomy, vol. 17, no. 6, pp. 565-569, 2012.

[18] M. Dehghan and F. Shakeri, "Solution of an integro-differential equation arising in oscillating magnetic fields using He's homotopy perturbation method," Progress in Electromagnetics Research, vol. 78, pp. 361-376, 2008.

[19] O. Abu Arqub, A. El-Ajou, A. S. Bataineh, and I. Hashim, "A representation of the exact solution of generalized LaneEmden equations using a new analytical method," Abstract and Applied Analysis, vol. 2013, Article ID 378593, 10 pages, 2013.

[20] M. K. Kadalbajoo and K. K. Sharma, "Numerical analysis of boundary-value problems for singularly-perturbed differential-difference equations with small shifts of mixed type," Journal of Optimization Theory and Applications, vol. 115, no. 1, pp. 145-163, 2002.

[21] M. K. Kadalbajoo and K. K. Sharma, "Numerical treatment of a mathematical model arising from a model of neuronal variability," Journal of Mathematical Analysis and Applications, vol. 307, no. 2, pp. 606-627, 2005.

[22] F. Mirzaee and S. F. Hoseini, "Solving singularly perturbed differential-difference equations arising in science and engineering with Fibonacci polynomials," Results in Physics, vol. 3, pp. 134-141, 2013.

[23] H. Xu and Y. Jin, "The asymptotic solutions for a class of nonlinear singular perturbed differential systems with time delays," The Scientific World Journal, vol. 2014, Article ID 965376, 7 pages, 2014

[24] F. Z. Geng, S. P. Qian, and M. G. Cui, "Improved reproducing kernel method for singularly perturbed differential-difference equations with boundary layer behavior," Applied Mathematics and Computation, vol. 252, pp. 58-63, 2015.

[25] Z. Sabir, H. A. Wahab, M. Umar, and F. Erdoğan, "Stochastic numerical approach for solving second order nonlinear singular functional differential equation," Applied Mathematics and Computation, vol. 363, p. 124605, 2019.

[26] J. K. Zhou, Differential Transformation and Its Applications for Electrical Circuits, Huazhong University Press, Wuhan, China, 1986.

[27] A. Arikoglu and I. Ozkol, "Solution of difference equations by using differential transform method," Applied Mathematics and Computation, vol. 174, no. 2, pp. 1216-1228, 2006.

[28] M. S. M. Selvi and L. Rajendran, "Application of modified wavelet and homotopy perturbation methods to nonlinear oscillation problems," Applied Mathematics and Nonlinear Sciences, vol. 4, no. 2, pp. 351-364, 2019.

[29] D. Ziane, M. H. Cherif, C. Cattani, and K. Belghaba, "Yanglaplace decomposition method for nonlinear system of local fractional partial differential equations," Applied Mathematics and Nonlinear Sciences, vol. 4, no. 2, pp. 489-502, 2019.

[30] M. Dewasurendra and K. Vajravelu, "On the method of inverse mapping for solutions of coupled systems of nonlinear differential equations arising in nanofluid flow, heat and mass transfer," Applied Mathematics and Nonlinear Sciences, vol. 3, no. 1, pp. 1-14, 2018.

[31] H. Chen, J. Jiang, D. Cao, and X. Fan, "Numerical investigation on global dynamics for nonlinear stochastic heat conduction via global random attractors theory," Applied Mathematics and Nonlinear Sciences, vol. 3, no. 1, pp. 175-186, 2018.

[32] A. T. El-Dessouky, "A note on strongly nonlinear parabolic variational inequalities," Applied Mathematics and Nonlinear Sciences, vol. 2, no. 2, pp. 443-448, 2017.

[33] M. C. Bortolan and F. Rivero, "Non-autonomous perturbations of a non-classical non-autonomous parabolic equation with subcritical nonlinearity," Applied Mathematics and Nonlinear Sciences, vol. 2, no. 1, pp. 31-60, 2017.

[34] M. Umar, F. Amin, H. A. Wahab, and D. Baleanu, "Unsupervised constrained neural network modeling of boundary value corneal model for eye surgery," Applied Soft Computing, vol. 85, p. 105826, 2019.

[35] M. Umar, Z. Sabir, and M. A. Z. Raja, "Intelligent computing for numerical treatment of nonlinear prey-predator models," Applied Soft Computing, vol. 80, pp. 506-524, 2019.

[36] M. A. Z. Raja, M. Umar, Z. Sabir, J. A. Khan, and D. Baleanu, "A new stochastic computing paradigm for the dynamics of nonlinear singular heat conduction model of the human head," The European Physical Journal Plus, vol. 133, no. 9, p. 364, 2018.

[37] Z. Sabir, M. A. Manzar, M. A. Z. Raja, M. Sheraz, and A. M. Wazwaz, "Neuro-heuristics for nonlinear singular Thomas-Fermi systems," Applied Soft Computing, vol. 65, pp. 152-169, 2018.

[38] M. A. Z. Raja, J. Mehmood, Z. Sabir, A. K. Nasab, and M. A. Manzar, "Numerical solution of doubly singular nonlinear systems using neural networks-based integrated intelligent computing," Neural Computing and Applications, vol. 31, no. 3, pp. 793-812, 2019.

[39] M. A. Z. Raja, Z. Sabir, N. Mehmood, E. S. Al-Aidarous, and J. A. Khan, "Design of stochastic solvers based on genetic algorithms for solving nonlinear equations," Neural Computing and Applications, vol. 26, no. 1, pp. 1-23, 2015. 